PROCEEDINGS OF THE

AMERICAN MATHEMATICAL SOCIETY

Volume 124, Number 12, December 1996, Pages 3897-3903

S 0002-9939(96)03497-1

\title{
CLASSIFYING SPACES AND HOMOTOPY SETS OF AXES OF PAIRINGS
}

\author{
KENSHI ISHIGURO
}

(Communicated by Thomas Goodwillie)

Dedicated to Professor Teiichi Kobayashi on his 60th birthday

\begin{abstract}
We consider the maps between classifying spaces of the form $B K \times$ $B L \rightarrow B G$. The main theorem shows that if the restriction map on $B L$ is a weak epimorphism, then the restriction on $B K$ should factor through the classifying spaces of the center of the compact Lie group $G$. An application implies that $B G$ is an $\mathrm{H}-$ space (Hopf space) if and only if $G$ is abelian.
\end{abstract}

For a map $f: Y \rightarrow Z$, the set of the homotopy classes of axes, denoted by $f^{\perp}(X, Z)$, consists of all homotopy classes of maps $\alpha: X \rightarrow Z$ such that there is a map (called a pairing) $\mu: X \times Y \rightarrow Z$ with restrictions (axes) $\left.\mu\right|_{X} \simeq \alpha$ and $\left.\mu\right|_{Y} \simeq f$, [16]. If $Z$ is an $\mathrm{H}$-space, then $f^{\perp}(X, Z)=[X, Z]$ : for the $\mathrm{H}$ multiplication $m: Z \times Z \rightarrow Z$, a pairing of $f$ and $\alpha$ is given by the composite map $m \circ(\alpha \times f)$. It follows that, for example, if $G, K$, and $L$ are compact Lie groups, then $f^{\perp}(K, G)=[K, G]$ with $f: L \rightarrow G$. In this paper we will study the classifying space version, namely $f^{\perp}(B K, B G)$ with $f: B L \rightarrow B G$. As group theoretical analog indicates, our results will show that few maps in $[B K, B G]$ belong to $f^{\perp}(B K, B G)$, in general. Other forms of axial maps (H-pairing) are studied in $[6]$.

The main theorem deals with the case that the map $f: B L \rightarrow B G$ is a weak epimorphism studied in [8]. (We recall the definition in §2.) An obvious example of a weak epimorphism is given by a map $f=B \rho$ induced by a group epimorphism $\rho$. The unstable Adams operations $\left\{\psi^{k}\right\}$ are also weak epimorphisms.

Theorem 1. Let $L$ and $G$ be connected compact Lie groups and let $K$ be a compact (not necessarily connected) Lie group. If $f: B L \rightarrow B G$ is a weak epimorphism, the following hold:

(1) If $\alpha \in f^{\perp}(B K, B G)$, then the map $\alpha$ factors through $B Z(G)$ up to homotopy, where $Z(G)$ denotes the center of $G$.

(2) Moreover, we have $f^{\perp}(B K, B G)=\operatorname{Hom}(K, Z(G))$.

Taking $L=G$ and $f=i d$ (the identity map), our problem asks about possible $B K$-actions on $B G$. In fact, this work was motivated by the following result of $\mathrm{G}$. Dula and D. Gottlieb.

Received by the editors April 10, 1995 and, in revised form, June 15, 1995.

1991 Mathematics Subject Classification. Primary 55R35; Secondary 55P15, 55P60.

Key words and phrases. Classifying space, mapping spaces, $p$-completion, Lie groups, weak epimorphism, pairing. 
Theorem 2 ([2]). Let $\alpha: X \rightarrow Z$ be a map, and $X$ an $H$-space. Then the following are equivalent.

(a) There exists a space $Y$ and a homotopy equivalence $X \times Y \rightarrow Z$ such that the orbit map $X \rightarrow X \times Y \rightarrow Z$ is homotopy equivalent to $\alpha$.

(b) The map $\alpha$ is the orbit of an action of $X$ on $Z$ and $\alpha^{\#}:[Z, X] \rightarrow[X, X]$ is onto.

For instance, let $X=B S^{1}$ and $Z=B U(n)$. A map $\alpha: B S^{1} \rightarrow B U(n)$ is induced by a homomorphism $\rho: S^{1} \rightarrow U(n)$ so that $\alpha=B \rho$. Since $B U(n)$ is indecomposable, [7], Theorem 2 implies that the $B S^{1}$-action on $B U(n)$ under the map $\alpha$ does not exist if $\alpha$ is induced by the inclusion $S^{1}=U(1) \hookrightarrow U(n)$. Theorem 1 says that the $B S^{1}$-action on $B U(n)$ under the map $\alpha$ exists if and only if $\rho\left(S^{1}\right)$ is central in $U(n)$. Generally, a consequence of Theorem 1 shows that $B K$-actions on $B G$ under $\alpha$ exist if and only if the map $\alpha: B K \rightarrow B G$ is induced by a homomorphism $\rho: K \rightarrow G$ such that $\rho(K)$ is central in $G$. In particular, if $K$ is connected and $G$ is semi-simple, there are no non-trivial $B K$-actions on $B G$.

Furthermore, if we take $K=L=G$ and $f=\alpha=i d$, the problem now asks whether $B G$ is an $\mathrm{H}$-space. Corollary 2.4 implies that $B G$ is an $\mathrm{H}$-space if and only if $G$ is abelian. ( $G$ need not be connected.) Related results were obtained in $[12]$ and $[1]$.

I would like to thank Giora Dula for bringing this problem to my attention. I would also like to thank my colleague, Nobuyuki Oda for his help.

\section{Mapping SPACES AND CENTRALIZERS OF $p$-TORAL GROUPS}

We give a necessary and sufficient condition that a map $\alpha: X \rightarrow Z$ be contained in $f^{\perp}(X, Z)$ in terms of mapping spaces. Then a special case of classifying space version is discussed.

Proposition 1.1. Suppose $X, Y$ and $Z$ are pointed connected spaces. For a map $f: Y \rightarrow Z$, a map $\alpha: X \rightarrow Z$ is contained in $f^{\perp}(X, Z)$ if and only if the map $f$ factors through $\operatorname{map}(X, Z)_{\alpha}$, the connected component of the mapping space containing $\alpha$, under the evaluation $\operatorname{map} \operatorname{map}(X, Z)_{\alpha} \stackrel{e v}{\longrightarrow} Z$.

Remark. It is easy to see that $\alpha \in f^{\perp}(X, Z)$ if and only if $f \in \alpha^{\perp}(Y, Z)$. Consequently $\alpha$ must factor through $\operatorname{map}(Y, Z)_{f}$.

Proof of Proposition 1.1. If $\alpha \in f^{\perp}(X, Z)$, there is a pairing $\mu: X \times Y \rightarrow Z$ with $\left.\mu\right|_{X} \simeq \alpha$ and $\left.\mu\right|_{Y} \simeq f$. Let $\bar{\mu}: Y \rightarrow \operatorname{map}(X, Z)_{\alpha}$ be the adjoint map of $\mu$. Then we see that the map $f$ is expressed as the composite of the adjoint map and the evaluation map :

$$
f: Y \stackrel{\bar{\mu}}{\longrightarrow} \operatorname{map}(X, Z)_{\alpha} \stackrel{e v}{\longrightarrow} Z
$$

since $e v \circ \bar{\mu}(y)=\bar{\mu}(y)(*)=\mu(*, y)=f(y)$.

Conversely, suppose $f$ factors through $\operatorname{map}(X, Z)_{\alpha}$ so that $f \simeq e v \circ \bar{\mu}$ for some map $\bar{\mu}: Y \rightarrow \operatorname{map}(X, Z)_{\alpha}$. Let $\epsilon: X \times \operatorname{map}(X, Z)_{\alpha} \rightarrow Z$ be the canonical map with $\epsilon(x, \beta)=\beta(x)$ for $x \in X$ and $\beta \in \operatorname{map}(X, Z)_{\alpha}$. Then a pairing is constructed as the composite

$$
\mu: X \times Y \stackrel{1_{X} \times \bar{\mu}}{\longrightarrow} X \times \operatorname{map}(X, Z)_{\alpha} \stackrel{\epsilon}{\longrightarrow} Z
$$


where $1_{X}$ is the identity map of $X$, since $\mu(x, y)=\epsilon \circ\left(1_{X} \times \bar{\mu}\right)(x, y)=\epsilon(x, \bar{\mu}(y))=$ $\bar{\mu}(y)(x)$ so that $\mu(x, *)=\bar{\mu}(*)(x)=\alpha(x)$ and $\mu(*, y)=\epsilon(*, \bar{\mu}(y))=e v \circ \bar{\mu}(y) \simeq$ $f(y)$.

We next consider the " $B G$ "-version. It is worth to recall some property of homomorphisms. Suppose $\rho: L \rightarrow G$ and $\alpha: K \rightarrow G$ are homomorphisms. If there is a pairing homomorphism $\mu: K \times L \rightarrow G$ with $\left.\mu\right|_{K}=\alpha$ and $\left.\mu\right|_{L}=\rho$, then the image $\rho(L)$ must be contained in the centralizer of $\alpha$ in $G$, denoted by $C_{G}(\alpha)$. The following is a " $B G$ "-analog at a prime $p$. If a map $\alpha: B K \rightarrow B G$ is induced by a homomorphism, let $C_{G}(\alpha)$ denote the centralizer of the homomorphism. For a $p$-toral group $K$ (a group extension of a torus by a finite $p$-group), it is known, [5] and [14], that any map $\alpha: B K \rightarrow B G$ (at $p)$ has the form $\alpha=B \eta \quad(\alpha=$ $\left.(B \eta)_{p}^{\wedge}\right)$ for some homomorphism $\eta$. Let $B G_{p}^{\wedge}$ denote the $p$-completion of $B G$. Since $\operatorname{map}(B K, B G)_{\alpha}$ is $p$-equivalent to $B C_{G}(\alpha)$, the following is immediate from Proposition 1.1.

Corollary 1.2. Suppose $K$ is p-toral. Then $\alpha \in f^{\perp}\left(B K_{p}^{\wedge}, B G_{p}^{\wedge}\right)$ if and only if the map $f$ factors through $B C_{G}(\alpha)_{p}^{\wedge}$ up to homotopy, under the map $B C_{G}(\alpha)_{p}^{\wedge} \rightarrow B G_{p}^{\wedge}$ induced by the inclusion.

As the above indicates, if the mapping space is computable, then the set of the homotopy classes of axes $f^{\perp}(X, Z)$ would be determined. It is, however, hard to compute $\operatorname{map}(X, Z)_{\alpha}$ or $\operatorname{map}(Y, Z)_{f}$ in general. Thus our work is to consider the problem, with or without calculation of mapping spaces.

\section{WEAK EPIMORPHisms AND THE PROOF OF THEOREM 1}

We will begin with some lemmas to prove Theorem 1.

Lemma 2.1. Let $R$ and $S$ be rings of polynomials in $n$ indeterminates over a field. If there is an epimorphsim $\varphi: R \rightarrow S$, then $\varphi$ is an isomorphsim.

Proof. Let $\mathfrak{p}=\operatorname{Ker} \varphi$ so that $R / \mathfrak{p} \cong S$. We need to show that $\mathfrak{p}=0$. The ideal $\mathfrak{p}$ is prime, since $R / \mathfrak{p}$ is an integral domain. Consequently, if $\operatorname{dim}(R)$ denotes the Krull dimension of $R$, then

$$
\operatorname{height}(\mathfrak{p})+\operatorname{dim}(R / \mathfrak{p})=\operatorname{dim}(R) .
$$

It follows that

$$
\begin{aligned}
\operatorname{dim}(S) & =\operatorname{dim}(R / \mathfrak{p}) \\
& =\operatorname{dim}(R)-\operatorname{height}(\mathfrak{p}) .
\end{aligned}
$$

Since $\operatorname{dim}(S)=\operatorname{dim}(R)=n$, we see that $h e i g h t(\mathfrak{p})=0$. In a domain, this means $\mathfrak{p}=0$.

Recall that, for any compact connected Lie group $G$, there is a covering $\gamma_{G} \rightarrow$ $\widetilde{G} \rightarrow G$ such that $\widetilde{G}=G_{s} \times T$ is a product of a simply-connected Lie group and a torus, and that $\gamma_{G}$ is a finite central subgroup of $\widetilde{G}$. Such a covering is called $a$ universal finite covering.

Lemma 2.2. Suppose $G$ is a connected compact Lie group and $H$ is a connected closed subgroup with inclusion $\iota: H \rightarrow G$. If the p-completed map $(B \iota)_{p}^{\wedge}$ : $(B H)_{p}^{\wedge} \rightarrow(B G)_{p}^{\wedge}$ is rationally equivalent, then $H=G$. 
Proof. Since $H$ and $G$ are connected, we can find universal finite coverings $\widetilde{H}$ and $\widetilde{G}$ so that we have the the commutative diagram

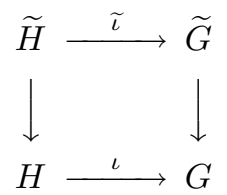

Note that $\widetilde{H}$ and $\widetilde{G}$ are products of simply-connected simple Lie groups and a torus, $\widetilde{H}=\prod_{i} \widetilde{H}_{i}$ and $\widetilde{G}=\prod_{j} \widetilde{G}_{j}$ respectively. Since the $\operatorname{map}(B \iota)_{p}^{\wedge}:(B H)_{p}^{\wedge} \rightarrow(B G)_{p}^{\wedge}$ is rationally equivalent, for each $\widetilde{G}_{j}$, by [8], we can find $\widetilde{H}_{i}$ such that the restricted $\operatorname{map}\left(B \widetilde{H}_{i}\right)_{p}^{\wedge} \rightarrow\left(B \widetilde{G}_{j}\right)_{p}^{\wedge}$ is a rational equivalence, and the map $(B \widetilde{\iota})_{p}^{\wedge}$ is diagonal. Using the classification of compact simply-connected simple Lie groups and their maximal rank subgroups, one can show that $\widetilde{\iota}\left(\widetilde{H}_{i}\right)=\widetilde{G}_{j}$. Hence $\widetilde{\iota}: \widetilde{H} \longrightarrow \widetilde{G}$ is onto. Consequently the inclusion $\iota: H \rightarrow G$ is an epimorphism, and therefore $H=G$.

Lemma 2.3. Let $G$ be a compact Lie group with center $Z(G)$. Then the map induced by the inclusion $i: Z(G) \hookrightarrow G$

$$
(B i)_{\#}:[B K, B Z(G)] \rightarrow[B K, B G]
$$

is one-to-one for any compact Lie group $K$.

Proof. Notice that $[B K, B Z(G)]=\operatorname{Hom}(K, Z(G))$, since $Z(G)$ is abelian, [11]. For $\rho_{1}, \rho_{2} \in \operatorname{Hom}(K, Z(G))$, suppose $(B i)_{\#}\left(B \rho_{1}\right)=(B i)_{\#}\left(B \rho_{2}\right)$. Thus, for each $p$-toral subgroup $K_{q}$ of $K$, we have $\rho_{1}(x)=\rho_{2}(x)$ for any $x \in K_{q}$, since the images of $\rho_{1}$ and $\rho_{2}$ are contained in the center $Z(G)$. Define $\rho \in \operatorname{Hom}(K, Z(G))$ by $\rho(k)=\rho_{1}(k) \rho_{2}(k)^{-1}$ for any $k \in K$ so that $\left.\rho\right|_{K_{q}}=0$ for any $p$-toral subgroup $K_{q}$. Since $\left[B K_{p}^{\wedge}, B G_{p}^{\wedge}\right]=\left[\underset{\operatorname{holim}}{\longrightarrow}\left(B K_{q}\right)_{p}^{\wedge}, B G_{p}^{\wedge}\right]=\operatorname{holim}\left[\left(B K_{q}\right)_{p}^{\wedge}, B G_{p}^{\wedge}\right]$, it follows that $\rho=0$. Consequently $\rho_{1}=\rho_{2}$, and therefore $B \rho_{1}=B \rho_{2}$.

Remark. For $\iota: H \hookrightarrow G$ the map $[B K, B H] \rightarrow[B K, B G]$ need not be one-toone. A counterexample is given by $\left[B \mathbb{Z} / 2, B T^{2}\right] \nrightarrow[B \mathbb{Z} / 2, B U(2)]$ where $T^{2}$ is a maximal torus of $U(2)$.

We consider $f^{\perp}(B K, B G)$ when $f: B L \rightarrow B G$ is a weak epimorphism, defined as follows. Suppose $L$ and $G$ are connected. A map $B L \rightarrow B G$ or $B L_{p}^{\wedge} \rightarrow B G_{p}^{\wedge}$ is called a weak epimorphism, [8], if there exists a fibration $Z \rightarrow B L \rightarrow B G$ or $Z \rightarrow B L_{p}^{\wedge} \rightarrow B G_{p}^{\wedge}$ such that $H^{*}(\Omega Z ; \mathbb{Q})$ is a finite dimensional $\mathbb{Q}-$ module or that $H^{*}\left(\Omega Z ; \mathbb{Z}_{p}^{\wedge}\right) \otimes \mathbb{Q}$ is a finite dimensional $\mathbb{Q}_{p}^{\wedge}$-module, respectively. Some examples were given in the introduction.

Proof of Theorem 1. First we show Part (1). Fix a prime $p$. Suppose $K_{q}$ is a $p$ toral subgroup of $K$ and $\alpha_{q}=\left.\alpha_{p}^{\wedge}\right|_{\left(B K_{q}\right)_{p}}$. Corollary 1.2 shows that the $p$-completed map $f_{p}^{\wedge}: B L_{p}^{\wedge} \rightarrow B G_{p}^{\wedge}$ factors through $B C_{G}\left(\alpha_{q}\right)_{p}^{\wedge}$ up to homotopy. Since $K_{q}$ is $p$-toral, $\pi_{0}\left(C_{G}\left(\alpha_{q}\right)\right)$ is a finite $p$-group. Since $B L_{p}^{\wedge}$ is 1 -connected, the map $f_{p}^{\wedge}$ factors through the $p$-completed classifying space of the connected component of the centralizer $C_{G}\left(\alpha_{q}\right)$. Since $f$ is a weak epimorphism, the map $f_{p}^{\wedge}$ is also a weak epimorphism. Consequently there is a fibration $Z \rightarrow B L_{p}^{\wedge} \rightarrow B G_{p}^{\wedge}$ satisfying 
certain finiteness conditions mentioned above. Let $H$ be the connected component of $C_{G}\left(\alpha_{q}\right)$ with inclusion $\iota: H \rightarrow G$. Then we have the commutative diagram

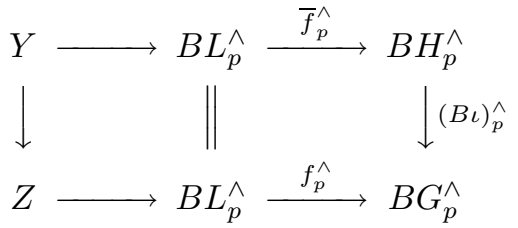

Here $Y$ is a homotopy fibre. Since $f_{p}^{\wedge}$ is a weak epimorphism, we see that $H^{*}\left(B L_{p}^{\wedge} ; \mathbb{Q}_{p}^{\wedge}\right) \cong H^{*}\left(B G_{p}^{\wedge} ; \mathbb{Q}_{p}^{\wedge}\right) \otimes H^{*}\left(Z ; \mathbb{Q}_{p}^{\wedge}\right)$. Thus, for the induced homomorphism $\left(f_{p}^{\wedge}\right)^{*}: H^{*}\left(B G_{p}^{\wedge} ; \mathbb{Q}_{p}^{\wedge}\right) \rightarrow H^{*}\left(B L_{p}^{\wedge} ; \mathbb{Q}_{p}^{\wedge}\right)$ there is a homomorphism (left inverse) $r: H^{*}\left(B L_{p}^{\wedge} ; \mathbb{Q}_{p}^{\wedge}\right) \rightarrow H^{*}\left(B G_{p}^{\wedge} ; \mathbb{Q}_{p}^{\wedge}\right)$ with $r \circ\left(f_{p}^{\wedge}\right)^{*}=1$. Consequently $r \circ\left(\bar{f}_{p}^{\wedge}\right)^{*} \circ\left((B \iota)_{p}^{\wedge}\right)^{*}=1$. Let $\varphi$ be the endomorphism $\left((B \iota)_{p}^{\wedge}\right)^{*} \circ r \circ\left(\bar{f}_{p}^{\wedge}\right)^{*}$ of the polynomial ring $H^{*}\left(B H_{p}^{\wedge} ; \mathbb{Q}_{p}^{\wedge}\right)$. Since $r \circ\left(\bar{f}_{p}^{\wedge}\right)^{*}$ is onto and $\left((B \iota)_{p}^{\wedge}\right)^{*}$ is one-to-one, the Krull dimension of the image of $\varphi$ is equal to $\operatorname{rank}(G)$. Hence the endomorphism $\varphi$ is an isomorphsim and therefore $\left((B \iota)_{p}^{\wedge}\right)^{*}$ is onto. Lemma 2.1 implies that $B H_{p}^{\wedge}$ is rationally equivalent to $B G_{p}^{\wedge}$. From Lemma 2.2 , we see that $C_{G}\left(\alpha_{q}\right)=G$. This implies that the map $\alpha_{q}$ is induced by a homomorphism into the center $Z(G)$.

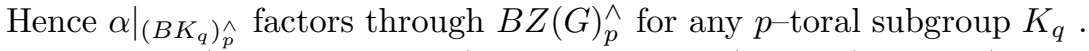

Since $B K_{p}^{\wedge} \simeq \underset{\operatorname{holim}}{\longrightarrow}\left(B K_{q}\right)_{p}^{\wedge}$, [9], the map $\alpha_{p}^{\wedge}: B K_{p}^{\wedge} \rightarrow B G_{p}^{\wedge}$ factors through $B Z(G)_{p}^{\wedge}$ for any $p$. Let $K_{0}$ denote the connected component of $K$ containing the identity so that there is an exact sequence $K_{0} \rightarrow K \stackrel{\pi}{\rightarrow} \pi_{0} K$. Consider the canonical fibration $B Z(G) \rightarrow B G \stackrel{B q}{\longrightarrow} B(G / Z(G))$. Since $\left(\left.B q \cdot \alpha\right|_{B K_{0}}\right)_{p}^{\wedge}$ is null homotopic, the map $\left.B q \cdot \alpha\right|_{B K_{0}}$ is rationally null homotopic. Since $K_{0}$ is connected, then $\left.B q \cdot \alpha\right|_{B K_{0}}=0$. This induces a map $\bar{\alpha}: B \pi_{0} K \rightarrow B(G / Z(G))$ with $B q \cdot \alpha=\bar{\alpha} \cdot B \pi$. Since $(B q \cdot \alpha)_{p}^{\wedge}=0$ for any $p$ and the homomorphism $\pi$ is onto, the restriction of $\bar{\alpha}$ on each $p$-Sylow subgroup of the finite group $\pi_{0} K$ is null homotopic. This implies $\bar{\alpha}=0$. Consequently $\alpha$ factors through $B Z(G)$.

Next we show Part (2). The canonical map $(B i)_{\#}:[B K, B Z(G)] \rightarrow[B K, B G]$ factors through $f^{\perp}(B K, B G)$. Let $\Phi$ be the map $[B K, B Z(G)] \rightarrow f^{\perp}(B K, B G)$ and let $\Psi$ be the map $f^{\perp}(B K, B G) \rightarrow[B K, B G]$ so that $(B i)_{\#}=\Psi \circ \Phi$. Part (1) shows $\Phi$ is onto. Lemma 2.3 shows $(B i)_{\#}$ is one-to-one. This implies $\Phi$ is one-toone. Thus $\Phi$ is bijective. Since $[B K, B Z(G)]=\operatorname{Hom}(K, Z(G))$, this completes the proof.

Remark. As shown in $\S 1$, there is a strong relationship between the set of the homotopy classes of axes $f^{\perp}(B K, B G)$ and $\operatorname{map}(B L, B G)_{f}$. Theorem 1 seems to indicate that $\operatorname{map}(B L, B G)_{f}$ can be homotopy equivalent to $B Z(G)$ when $f: B L \rightarrow B G$ is a weak epimorphism. One can show, however, that $\operatorname{map}\left(B S^{3}, B S^{3}\right)_{\psi^{k}} \nsucceq B Z\left(S^{3}\right)$ (without $p$-completion), from the following result of Dwyer-Mislin [3] : The space of pointed maps $\operatorname{map}_{*}\left(B S^{3}, B S^{3}\right)_{\psi^{k}}$ is homotopy equivalent to Sullivan's profinite completion $S O(3)^{\wedge}$. What to ask is the $p$-equivalence between $\operatorname{map}(B L, B G)_{f}$ and $B Z(G)$. A result of Jackowski-McClure-Oliver [9] and Notbohm [15] shows that if $f: B G \rightarrow B G$ is a self-equivalence, the $\operatorname{map} B Z(G) \rightarrow \operatorname{map}(B G, B G)_{f}$ is a $\bmod p$ equivalence for any prime $p$. A related result can be found in [4].

Corollary 2.4. Suppose $G$ is a compact (not necessarily connected) Lie group. If $B G$ is an $H$-space, then $G$ is abelian. 
Proof. If $B G$ is an $\mathrm{H}$-space, then $(i d)^{\perp}(B G, B G)=[B G, B G]$. Suppose first that $G$ is connected. Taking $\alpha=i d$ in Theorem 1 , we see that the identity map of $B G$ factors through $B Z(G)$. Consequently $G=Z(G)$, and therefore $G$ is a torus. In general, there is the exact sequence $G_{0} \rightarrow G \rightarrow \pi_{0} G$. If $B G$ is an $\mathrm{H}-$ space, from the fibration $B G_{0} \rightarrow B G \rightarrow B \pi_{0} G$ it follows that both $B G_{0}$ and $B \pi_{0} G$ are also $\mathrm{H}$-spaces. This implies that the groups $G_{0}$ and $\pi_{0} G$ are abelian, since $\pi_{0} G$ is a finite group so that $B \pi_{0} G=K\left(\pi_{0} G, 1\right)$. Let $\pi=\pi_{0} G$ and let $\pi_{p}$ denote a $p$-Sylow subgroup of the finite group $\pi$. Suppose $G_{p}$ is the subgroup of $G$ over $\pi_{p}$ :

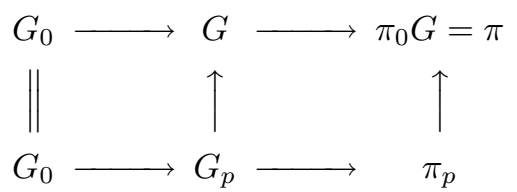

Since $B G$ is an $\mathrm{H}$-space, the space $B G_{p}$ is also an $\mathrm{H}$-space. Notice that $G_{p}$ is $p$ toral. Consequently the $\mathrm{H}-$ multiplication on $B G_{p}$ is induced by a homomorphism $G_{p} \times G_{p} \rightarrow G_{p}$. This implies that $G_{p}$ is abelian for any prime $p$, and therefore $G\left(=G_{0} \times \pi_{0} G\right)$ is abelian.

Remark. The $p$-completed version of Corollary 2.4 does not hold. For instance, the symmetric group $\Sigma_{3}$ is non-abelian, but the 2 -completed classifying space $\left(B \Sigma_{3}\right)_{2}^{\wedge}=B \mathbb{Z} / 2$ is an $\mathrm{H}-$ space.

\section{REFERENCES}

1. J. Aguadé and L. Smith, On the mod p torus theorem of John Hubbuck, Math. Z. 191 (1986), 325-326. MR 87e:57044

2. G. Dula and D. Gottlieb, Splitting off H-spaces and Conner-Raymond Splitting Theorem, J. Fac. Sci. Univ. Tokyo Sect. IA Math. 37 (1990), 321-334. MR 91i:55018

3. W.G. Dwyer and G. Mislin, On the homotopy type of the components of $\operatorname{map}_{*}\left(B S^{3}, B S^{3}\right)$, Proc. 1986 Barcelona conference, LNM 1298 (1987), 82-89. MR 89e:55018

4. W.G. Dwyer and C. Wilkerson, The center of a p-compact group, Preprint.

5. W.G. Dwyer and A. Zabrodsky, Maps between classifying spaces, Proc. 1986 Barcelona conference, LNM 1298 (1987), 106-119. MR 89b:55018

6. Y. Hemmi, The projective plane of an H-pairing, J.P.A.A. 75 (1991), 277-296. MR 92m:55011

7. K. Ishiguro, A p-local splitting of $B U(n)$, Proc. of AMS 95(2) (1985), 307-311. MR 86m:55023

8. K. Ishiguro and D. Notbohm, Fibrations of classifying spaces, Transaction of AMS 343(1) (1994), 391-415. MR 94h:55028

9. S. Jackowski, J.E. McClure and B. Oliver, Homotopy classification of self-maps of BG via G-actions Part I and Part II, Ann. of Math. 135 (1992), 183-226, 227-270. MR 93e:55019

10. S. Jackowski, J.E. McClure and B. Oliver, Self homotopy equivalences of classifying spaces of compact connected Lie groups, Preprint.

11. R. Lashof, P. May and G. Segal, Equivariant bundles with abelian structural group, Contemporary Math. 19 (1983), 167-176. MR 85b:55023

12. J. Lin, A cohomological proof of the torus theorem, Math. Z. 190 (1985), 469-476. MR 87c:55009

13. G. Mislin and J. Thévenaz, The $Z^{*}-$ Theorem for compact Lie groups, Math. Ann. 291 (1991), 103-111. MR 92i:22006

14. D. Notbohm, Maps between classifying spaces, Math. Z. 207 (1991), 153-168. MR 92b:55017

15. D. Notbohm, Maps between classifying spaces and apllications,, J.P.A.A. 89 (1993), 273-294. MR 95c:55019 
16. N. Oda, The homotopy set of the axes of pairings, Canad. J. Math. 42 (1990), 856-868. MR 91i:55019

17. N. Oda, Localization of the homotopy set of the axes of pairings, Adams memorial symposium on algegraic topology volume 1, London Math. Soc. Lecture Notes 175, Cambridge Univ. Press (1992), 163-177. MR 93i:55014

Department of Mathematics, Fukuoka University, Fukuoka 814-80, Japan

E-mail address: kenshi@ssat.fukuoka-u.ac.jp 